\title{
Avaliação da dor e incapacidade funcional em pacientes com lombalgia crônica submetidos a um programa educativo de uma "escola de coluna"
}

\author{
Evaluation of pain and functional disability in patients with chronic low back pain submitted \\ to an educational program of a "spine school"
}

Evaluación del dolor y la discapacidad funcional en pacientes con dolor lumbar crónico sometidos a un programa educativo de una "escuela de la columna vertebral"

Djanira Florentino Silva ${ }^{1 *}$, Lucas Pereira Balbino ${ }^{1}$, Marcilene Glay Viana Pessoa ${ }^{1}$, Maria Jasmine Gomes da Silva' ${ }^{1}$, Andreza Faro de Oliveira' ${ }^{1}$, Paulo José Medeiros de Souza Costa ${ }^{1}$.

\section{RESUMO}

Objetivo: Avaliar o nível de intensidade da dor lombar e o índice de incapacidade funcional, antes e após intervenção, através do Programa Educativo de uma "Escola de Coluna", em pacientes com lombalgia crônica. Métodos: Estudo com abordagem quantitativa do tipo interventiva longitudinal, realizado em um serviço público de fisioterapia, com 24 pacientes com diagnóstico de lombalgia crônica. A Escala Visual Analógica da Dor (EVA) e o Questionário Oswestry Disability Index (ODI) foram aplicados em dois momentos, na avaliação inicial- antes da intervenção; e na avaliação final- após a intervenção. O programa deste estudo é composto por 10 atividades, com duração de 60 minutos cada, com frequência de duas vezes por semana. $O$ teste $t$ pareado foi aplicado para identificar diferenças pré e pós-intervenção $(p \leq 0,05)$. Resultados: A análise dos dados revelou melhora significativa na intensidade da dor lombar, quando comparado pré e pós-intervenção $(p<0,0001)$ e aumento na capacidade funcional comparando as avaliações inicial e final $(p<0,001)$. Conclusão: A abordagem educativa e terapêutica, proposta desta "Escola de Coluna", apresentou resultados relevantes quanto à melhora da dor e da capacidade funcional em lombalgia crônica.

Palavras-chave: Dor lombar, Educação em saúde, Fisioterapia.

\begin{abstract}
Objective: To evaluate the level of intensity of low back pain and the index of functional disability, before and after intervention, through the Educational Program of a "Spine School", in patients with chronic low back pain. Methods: Study with a quantitative longitudinal intervention approach, carried out in a public physiotherapy service, with 24 patients diagnosed with chronic low back pain. The Visual Analog Pain Scale (VAS) and the Oswestry Disability Index Questionnaire (ODI) were applied in two moments, in the initial assessment - before the intervention; and in the final evaluation - after the intervention. The program of this study consists of 10 activities, lasting 60 minutes each, twice a week. The paired t-test was applied to identify differences before and after intervention $(p \leq 0.05)$. Results: Data analysis revealed a significant improvement in the intensity of low back pain, when compared before and after the intervention $(p<0.0001)$ and an increase in functional capacity comparing the initial and final evaluations $(p<0.001)$. Conclusion: The educational and therapeutic approach, proposed by this "Column School", presented relevant results regarding the improvement of pain and functional capacity in chronic low back pain.
\end{abstract}

Keywords: Low back pain, Health education, Physical therapy specialty.

1 Universidade de Ciências da Saúde de Alagoas (UNCISAL), Maceió - AL.
${ }^{\star}$ E-mail: djaniraflorentino@bol.com.br

SUBMETIDO EM: 6/2020

ACEITO EM: 7/2020

PUBLICADO EM: 11/2020 


\section{RESUMEN}

Objetivo: Evaluar el nivel de intensidad del dolor lumbar y el índice de discapacidad funcional, antes y después de la intervención, a través del Programa Educativo de una "Escuela de la columna vertebral", en pacientes con dolor lumbar crónico. Métodos: Estudio con un enfoque intervencionista longitudinal cuantitativo, realizado en un servicio público de fisioterapia, con 24 pacientes diagnosticados de dolor lumbar crónico. La Escala Visual Analógica del Dolor (VAS) y el Cuestionario del Índice de Discapacidad de Oswestry (ODI) se aplicaron en dos momentos, en la evaluación inicial, antes de la intervención; y en la evaluación final, después de la intervención. El programa de este estudio consta de 10 actividades, con una duración de 60 minutos cada una, dos veces por semana. La prueba t pareada se aplicó para identificar diferencias antes y después de la intervención $(p \leq 0.05)$. Resultados: El análisis de datos reveló una mejora significativa en la intensidad del dolor lumbar, en comparación antes y después de la intervención $(p<0,0001)$ y un aumento en la capacidad funcional comparando las evaluaciones iniciales y finales $(p<0,001)$. Conclusión: El enfoque educativo y terapéutico, propuesto por esta "Escuela de Columna", presentó resultados relevantes con respecto a la mejora del dolor y la capacidad funcional en el dolor lumbar crónico.

Palabras clave: Dolor de la región lumbar; Educácion en salud, Fisioterapia.

\section{INTRODUÇÃO}

A lombalgia é uma condição clínica caracterizada por dor e limitação funcional na região lombar, com ou sem irradiação para os membros inferiores (ALMEIDA DC e KRAYCHETE DC, 2017; FERREIRA A, et al., 2017). É um dos sintomas musculoesqueléticos mais comuns no mundo, podendo atingir cerca de $80 \%$ da população, em algum período da vida, sobretudo acima dos 40 anos e em fase produtiva, acometendo mais mulheres do que homens (POLLI GB, et al., 2018; HAEFFNER R, et al., 2015).

A dor lombar é responsável pelas principais causas de absenteísmo ao trabalho, incapacidade e aposentadoria precoce (STEFANE S e MARINOVIC H, 2013; IGUTI AM, et al., 2015). Sua origem é multifatorial, podendo ser decorrente de causas: degenerativas, inflamatórias, infecciosas, tumorais, mecânico-posturais, uso inadequado da coluna vertebral durante as atividades do cotidiano, entre outras (GAIDESKI F, et al., 2019).

De acordo com o tempo de acometimento, a lombalgia é classificada em três fases: aguda - duração menor que seis semanas; sub-aguda - entre seis e doze semanas; e fase crônica - quando é superior a três meses (BARRETO JM, et al., 2019). Aproximadamente 15\% da população acometida por esta desordem desenvolve cronicidade (VALENÇA JBM e ALENCAR MCB, 2018).

Esta afecção é responsável pelo uso frequente dos serviços de saúde, sendo uma das principais causas de licenças médicas, resultando em elevados custos e despesas em seu tratamento (NASCIMENTO JM, et al., 2015). Diante deste contexto, surgiu a "Back School". Um recurso terapêutico diferenciado, que atua na prevenção, cuidados e tratamento das algias da coluna vertebral (ANDRADE SC, et al., 2005).

A "Back School" teve sua origem na Suécia, em 1969, desenvolvida pela fisioterapeuta Mariane Zachrisson-Forssell. Trata-se de um programa educativo e terapêutico postural, com informações teóricoeducativas e exercícios terapêuticos, especificamente, para a coluna e membros inferiores, direcionado a indivíduos com algias na coluna com o propósito de ensinar e orientar sobre postura, capacitando-os a se protegerem ativamente de lesões (TSUKIMOTO GR, et al., 2006).

No Brasil, a "Escola de Postura" (Back School) iniciou em 1972, por Knoplich, no Hospital do Servidor Público Estadual de São Paulo (CHUNG TM, 1996; ANDRADE SC, et al., 2005). Desde então, têm sido utilizadas, diversas nomenclaturas para esse programa, como: Escola Postural, Escola de Coluna, Programa de Educação Postural, entre outras (NOLL M, et al., 2014). No presente estudo foi utilizado o termo "Escola de Coluna". 
Estima-se que no Brasil, 10 milhões de brasileiros são afastados do trabalho devido à dor lombar (SPONCHIADO P e CARVALHO AR, 2007). Esta traduz-se como um relevante problema de saúde pública, comum nas sociedades industrializadas, com repercussão negativa na vida familiar e sócio-econômica, comprometendo a realização das atividades laborais, resultando em limitações que afetam diretamente a qualidade de vida, aumentando com isso a procura por tratamento no intuito de minimizar os prejuízos causados pela lombalgia crônica (JUNIOR MH, et al., 2010; SILVA JCA, et al., 2014).

Desta forma, considerando o impacto social desta síndrome, este estudo objetivou avaliar o nível de intensidade da dor lombar e o índice de incapacidade funcional, antes e após intervenção, através do Programa Educativo e Terapêutico de uma "Escola de Coluna", em pacientes com lombalgia crônica.

\section{MÉTODOS}

Trata-se de um estudo com abordagem quantitativa do tipo interventiva longitudinal, realizado em um serviço público de Fisioterapia, no período de agosto a dezembro de 2018. O presente estudo foi aprovado no Comitê de Ética em Pesquisa da Universidade Estadual de Ciências da Saúde de Alagoas (UNCISAL), sob o no 2.728.636, CAAE: 88982518.8.0000.5011. De acordo com a resolução 466/12 do Conselho Nacional de Saúde, todos os participantes assinaram o Termo de Consentimento Livre e Esclarecido (TCLE). Este serviço desenvolve um Programa de "Escola de Coluna" para os pacientes que são encaminhados com algias na coluna vertebral. Este programa é uma iniciativa de educação em saúde que faz parte do conteúdo prático da disciplina de Saúde do Adulto e do Trabalhador II, junto aos discentes do 4ํㅡㄹ Ano do Curso de Fisioterapia da UNCISAL.

A amostra foi composta por 33 pacientes com diagnóstico de lombalgia crônica, que faziam parte da lista de espera deste serviço. Os critérios de inclusão foram: pacientes com dor lombar crônica, de ambos os sexos e faixa etária entre 20 e 60 anos de idade. E os critérios de exclusão, foram pacientes com: fibromialgia, fraturas e/ou cirurgia na coluna vertebral, processo tumoral, doenças inflamatórias como artrite reumatóide, e infecciosas da coluna vertebral. A amostra foi determinada por conveniência e a amostragem foi censitária. O percurso desse estudo foi desenvolvido em três etapas: avaliação inicial, e avaliação final, após a intervenção.

$\mathrm{Na}$ avaliação inicial foi realizada a coleta de dados através de formulário, para registrar informações do prontuário como: dados sócio-demográficos (idade, sexo, nível de escolaridade, estado civil, ocupação), e em seguida foram aplicados os instrumentos para avaliação da dor e incapacidade funcional em lombalgia.

Afim de identificar o nível de intensidade da dor lombar referida pelo paciente, foi utilizada a Escala Visual Analógica da Dor (EVA). Esta é semelhante a uma régua numerada de 0 a 10 , sendo um instrumento importante para avaliar a evolução do paciente durante o tratamento de maneira mais fidedigna, de acordo com o grau de melhora ou piora da dor (TOBO A, et al., 2010). Onde "0" significa ausência total de dor; e "10" o nível de dor máxima suportável pelo paciente.

Para avaliar o índice de incapacidade funcional em lombalgia, foi utilizado como instrumento o Questionário Oswestry Disability Index (ODI), validado para o português por Vigatto R, et al. (2007). É um questionário auto-administrado, composto por 10 seções com perguntas relacionadas aos temas: intensidade da dor, cuidados pessoais, levantamento de peso, andar, permanecer sentado, permanecer em pé, sono, atividade sexual, vida social e viagem. A pontuação vai de 0 (sem incapacidade funcional) a 100 (totalmente incapacitado) (FAIRBANK JC e PYNSENT PB, 2000). A escala EVA e o questionário ODI foram aplicados em dois momentos, na avaliação inicial- antes da intervenção; e na avaliação final - após a intervenção.

Após a avaliação inicial, os participantes foram encaminhados à segunda etapa- Intervenção (programa de uma "Escola de Coluna"). A estratégia da Escola de Coluna deste estudo, com base em outras escolas no Brasil, estruturou-se com enfoque educativo através de aulas teórico-práticas visando ensinar aos participantes sobre as posturas adequadas na realização das AVD's (atividades de vida diária) e das AVP's (atividades de vida profissional); e abordagem terapêutica através de uma série de exercícios de alongamento e fortalecimento muscular, para melhora da força, flexibilidade e relaxamento. Este programa é composto por 10 atividades, com duração de 60 minutos cada, com frequência de duas vezes por semana, durante um período de 5 semanas. 
As aulas teórico-práticas abordaram temas como: noções de anatomia da coluna vertebral; biomecânica da coluna vertebral; doenças da coluna vertebral; fatores causadores da dor lombar; postura e equilíbrio; noções de ergonomia - cuidados ergonômicos; medidas de proteção articular e movimentos corretos para a coluna; treinamento e modificação de posturas nas AVD's e nas AVPs; cuidados em situações de crise; importância e benefícios dos exercícios para manutenção de uma coluna saudável e práticas esportivas apropriadas para a coluna.

Estas atividades foram aulas do tipo exposição dialogada teórico/prática, enfatizando a troca de conhecimentos e discussão do tema em estudo e vivência de posturas adequadas para facilitar a realização das AVD's.

Os participantes eram convidados a demonstrar, de forma prática, os movimentos e atitudes quanto ao sentar, levantar, deitar, pegar objetos no chão, carregar peso, agachar, varrer a casa e permanecer em pé. Depois disto, eles realizavam esses movimentos e posturas de acordo com as orientações recebidas, repetindo-os, até a compreensão dos mesmos, visando também promover maior interação entre os participantes do estudo e a equipe da Escola.

Durante as aulas foi dada atenção especial ao vocabulário simples e de fácil compreensão aos integrantes da pesquisa, utilizando-se recursos áudio-visuais (computador e projetor multimídia) e peças anatômicas da coluna vertebral e suas estruturas, com o objetivo de tornar mais prático e compreensível os conteúdos durante os encontros.

Na sequência, após as aulas teórico-práticas, foram realizados exercícios terapêuticos de alongamento e fortalecimento das cadeias musculares anterior - posterior do tronco e membros inferiores; treino respiratório; posturas de relaxamento; orientações e treinamento da postura para as AVD's, como: sentar, levantar, deitar, andar, manter-se em pé, pegar objetos no chão e levantamento de peso. Foi enfatizada a necessidade da manutenção do programa de exercícios domiciliares e aplicação das orientações quanto a maneira correta para a realização das AVDs e AVPs.

Após a décima aula, foi realizada reavaliação (terceira etapa- após intervenção), onde foram utilizados os instrumentos da avaliação inicial (anterior à intervenção), com o objetivo de avaliar a evolução dos pacientes após a aplicação do programa "Escola de Coluna".

Para análise estatística foi utilizado o programa Bioestat®, versão 5.0. A análise dos dados quantitativos obtidos com a aplicação dos instrumentos EVA e ODI, foi realizada utilizando-se estatística descritiva. Foi feita estatística inferencial, para comparar as variáveis intensidade de dor e capacidade funcional, nos momentos pré e pós-intervenção, tendo sido utilizado o teste t pareado para amostras dependentes. Adotouse o nível de significância de $p \leq 0,05$.

\section{RESULTADO}

Dos 33 pacientes que iniciaram o programa da Escola de Coluna, desenvolvido em três etapas: Préintervenção, Intervenção e Pós-Intervenção, nove (9) deles não concluíram as dez aulas por motivos diversos: quatro (4) deixaram de vir sem justificativa, três (3) ficaram doentes, e dois (2) não conseguiram liberação do trabalho.

Dessa forma, os resultados foram analisados a partir de uma amostra de 24 pacientes com dor lombar crônica, de ambos os sexos. O sexo feminino foi predominante $(70,83 \%), 50 \%$ dos indivíduos tinham idade entre 50-59 anos e a maioria dos participantes eram casados (62,50\%), 33,34\% não tinham concluído o ensino fundamental, e 37,50\% encontravam-se afastados de suas atividades laborais.

A Tabela 1 ilustra os resultados obtidos antes e após a intervenção, em relação ao nível de intensidade da dor. Os valores médios de intensidade da dor relatados pelos participantes do estudo, aplicando-se a escala EVA, caíram de 6,20 (antes) para 2,45 (depois), com nível de significância estatística $(p<0,0001)$. 
Tabela 1 - Evolução do nível de intensidade da dor (EVA) dos participantes da pesquisa antes e após a aplicação do Programa da Escola de Coluna.

\begin{tabular}{ccccccc}
\hline \multirow{2}{*}{ Testes } & \multirow{N}{*}{} & \multicolumn{2}{c}{ Antes } & \multicolumn{2}{c}{ Depois } & p \\
\cline { 2 - 6 } & & Média & DP & Média & DP & Unilateral \\
\hline EVA & 24 & 6,20 & 2,75 & 2,45 & 2,06 & $\mathrm{p}<0,0001$ \\
\hline
\end{tabular}

Fonte: Silva DF, et al., 2020.

A Tabela 2 apresenta os resultados obtidos antes e após a intervenção, em relação ao índice de incapacidade funcional decorrente da lombalgia. Observa-se que houve redução deste índice, demonstrando uma melhora funcional dos participantes. Foi utilizado o questionário Oswestry Disability Index, havendo queda da média de 39,00 (antes) para 30,95 (depois) após a aplicação do programa Escola de Coluna, com um nível de significância estatística de $p<0,001$.

Tabela 2 - Evolução do índice de incapacidade funcional (ODI) dos participantes da pesquisa antes e após a aplicação do Programa da Escola de Coluna.

\begin{tabular}{|c|c|c|c|c|c|c|}
\hline \multirow{2}{*}{ Testes } & \multirow{2}{*}{$\mathbf{N}$} & \multicolumn{2}{|c|}{ Antes } & \multicolumn{2}{|c|}{ Depois } & \multirow{2}{*}{$\begin{array}{c}\mathbf{p} \\
\text { Unilateral }\end{array}$} \\
\hline & & Média & DP & Média & DP & \\
\hline ODI & 24 & 39,00 & 12,54 & 30,95 & 12,76 & $p<0,0010$ \\
\hline
\end{tabular}

Fonte: Silva DF, et al., 2020.

\section{DISCUSSÃO}

Entre as condições dolorosas que afetam a população, a dor lombar tem sido ultrapassada apenas pela cefaleia (ANDRADE SC, et al., 2005). A intensidade da lombalgia é relevante, devido à alta prevalência e ao custo elevado de seu tratamento, comprometendo a funcionalidade e a qualidade de vida dos indivíduos, diminuindo, assim, a produtividade nas tarefas de casa e do trabalho, causando problemas psicológicos e mudanças de comportamento (SALVETTI MG e PIMENTA CAM, 2007; OLIVEIRA ES, et al., 2004).

Dentre as causas que mais contribuem para recidivas desse quadro doloroso, está o uso inadequado da coluna vertebral durante as tarefas do cotidiano, tornando relevante e indispensável a intervenção preventiva e educativa nesta condição (FERREIRA A, et al., 2017; ANDRADE SC, et al., 2005). Dessa forma, no processo de reabilitação da lombalgia, o método Escola de Coluna destaca-se como recurso importante na assistência aos pacientes com dor lombar, alinhando tratamento e prevenção, enfatizando a aplicação de materiais educativos e orientações sobre os riscos que comprometem a função e aumentam a dor, devido à inatividade e posturas inadequadas (TSUKIMOTO GR, et al., 2006).

O programa de Escola da Coluna do presente estudo promoveu melhora significativa na intensidade da dor lombar, quando comparado pré e pós-intervenção $(p<0,0001)$. Em outro estudo, Rodrigues GJT e Garcia CA (2017) avaliaram a dor por meio da Escala EVA em pacientes com lombalgia crônica, também apresentando em seus resultados diminuição do quadro álgico após intervenção $(p=0,039)$.

Outras pesquisas contribuem com os dados do presente estudo, apresentando resultados eficazes na gestão da dor. Scheuchuk C e Comerlato T (2017), avaliaram os efeitos da educação em saúde como parte do tratamento fisioterapêutico, comparando os resultados da intervenção fisioterapêutica de forma isolada e associada à educação em saúde por meio de doze sessões. Constataram que houve diferença estatisticamente significativa na comparação do valor da variável dor (EVA) antes e após o tratamento para ambos os grupos. Porém na avaliação dos resultados de cada grupo isoladamente, observou-se uma melhora superior no grupo que recebeu tratamento fisioterapêutico associado à educação em saúde.

Em um programa de doze sessões de cinesioterapia aliado à educação postural, com frequência de uma vez por semana, e duração de três meses, observou-se diminuição da dor $(p<0,05)$ utilizando-se a escala EVA (KORELO RIG, et al., 2013).

Vários outros estudos apresentaram redução da dor nos pacientes submetidos a variados programas de Escolas de Coluna (SAHIN N, et al., 2011; YANG EJ, et al., 2010; MARTINS MRI, et al., 2010; ANDRADE 
SC, et al., 2008; SHIRADO O, et al., 2005). No entanto, nenhum deles mostrou a efetividade com o nível de significância $(p<0,0001)$ encontrado neste estudo.

A evolução positiva no nível de intensidade da dor com o programa Escola de Coluna pode ser decorrente dos exercícios terapêuticos realizados, os quais auxiliam na redução do quadro álgico e consequentemente na melhora da função, como também da adoção de posturas adequadas para a coluna vertebral, a partir da compreensão e conscientização das orientações por parte dos pacientes (SILVA JCA, et al., 2014).

As atividades laborais são diretamente prejudicadas pela lombalgia crônica, devido a incapacidade funcional, perda significativa da qualidade de vida e consequências socioeconômicas (SALVETTI MG, et al., 2012; HELFENSTEIN MJ, et al., 2010; HOY D, et al., 2010).

$\mathrm{Na}$ atual pesquisa os participantes apresentaram um relevante aumento na capacidade funcional (conforme o índice de Incapacidade de Oswestry- ODI) comparando as avaliações inicial e final $(p<0,001)$. Ferreira MS e Navega MT (2010), encontraram também resultados favoráveis em seu estudo com 41 pacientes que participaram de um programa de Escola Postural de seis encontros, e Tobo A, et al. (2010) em estudo com amostra de 43 pacientes.

Outro estudo que avaliou a eficácia do programa Back School na lombalgia e incapacidade funcional de enfermeiros iranianos, apresentou redução da dor lombar e da incapacidade funcional no grupo de intervenção comparado ao grupo controle. Os autores concluíram que este programa pode ser adequado para prevenir a dor e a incapacidade funcional entre enfermeiros que trabalham em ambientes hospitalares (PAKBAZ M, et al., 2019)

A resposta positiva para a funcionalidade, evidenciada após a aplicação do programa de Escola de Coluna, pode ter sido alcançada em virtude da melhora da dor, da realização de exercícios durante o período de intervenção, e das orientações para a execução adequada de AVDs, que possivelmente levaram os participantes ao replanejamento das atividades e mudanças de hábitos posturais durante a execução de suas funções (FERREIRA MS e NAVEGA MT, 2010; BARTZ PT, et al., 2015).

Arcanjo GN, et al. (2008) em sua pesquisa, referiram a influência dos exercícios físicos (alongamento) na melhora da flexibilidade, da conscientização postural e da ergonomia, indicando que estes possivelmente contribuíram no alívio do quadro álgico. O exercício é uma atividade física importante na prevenção e reabilitação de indivíduos com lombalgias, sendo indicado para manutenção e melhora da flexibilidade, força e função muscular, condicionamento físico, bem-estar físico e mental, além de reduzir a dor lombar (SILVA PHB, et al., 2013; MACEDO CSG, et al., 2010).

Em estudo de revisão bibliográfica, sobre a história e a aplicação da estratégia de educação em saúde Escola de Coluna, na lombalgia crônica, os autores concluíram que esta foi eficaz para melhora dos quadros de dor e incapacidade funcional em curto prazo, comparada a outros tratamentos convencionais (exercícios cinesioterapêuticos e manipulações espinhais) (ANDRADE SC, et al., 2005).

Noll M, et al. (2014) em sua revisão, tiveram como objetivo identificar os estudos sobre as Escolas Posturais desenvolvidas no Brasil. Estas Escolas apresentaram diferenças, tanto nas propostas de intervenções quanto no desenho metodológico. Mas, através da utilização de instrumentos semelhantes (avaliação da intensidade da dor, da capacidade funcional e da qualidade de vida) entre os estudos quantitativos, foi possível realizar uma comparação parcial da eficácia desses programas.

De uma forma geral, os estudos demonstraram efetividade na diminuição da dor, melhora da capacidade funcional e da qualidade de vida, porém, observou-se resultados contraditórios em relação à eficácia a médio e longo prazo. Logo, os programas de Escola de Coluna são amplamente utilizados, mas existem algumas dificuldades em determinar a eficácia dos mesmos devido a variação na metodologia e nas formas de avaliação utilizadas (DI FABIO RP, 1995).

O grande diferencial encontrado nos resultados deste estudo foi a relevância da significância estatística quanto a redução da intensidade da dor e da melhora da capacidade funcional, quando comparada aos outros estudos da literatura. Apesar de não ter sido feito a comparação direta de cada passo da execução da Escola 
de Coluna da UNCISAL com as aplicadas em outros serviços, pode-se inferir que o modelo de 10 sessões com aulas teórico-práticas, do tipo exposição dialogada, pode ser um grande diferencial para a obtenção destes resultados. Para Chung TM (1996), apesar dos diversos programas de Escola de Coluna existentes, os melhores resultados são alcançados quando o paciente se torna um gerenciador da sua saúde.

As limitações do atual estudo foram: a ausência de um grupo controle em que se comparasse a Escola de Coluna com as aulas e o mesmo tratamento sem as aulas (fisioterapia tradicional), e o acompanhamento dos participantes por um prazo maior. Portanto, é relevante dar continuidade com um número maior de participantes, inclusão de grupo controle e avaliação da efetividade do programa a médio e longo prazo.

\section{CONCLUSÃO}

A abordagem educativa e terapêutica, proposta desta "Escola de Coluna", apresentou resultados relevantes quanto à melhora da dor e da capacidade funcional em lombalgia crônica. Demonstrando que este programa, através das aulas teórico-práticas e dos exercícios, com intervenção para orientações posturais nas tarefas do cotidiano, fundamento principal deste, mostrou eficácia no modelo como foi executado. Acredita-se que estes resultados serão úteis na realização de trabalhos futuros, sugerindo a continuidade de estudos a longo prazo para avaliar a efetividade de programas semelhantes.

\section{REFERÊNCIAS}

1. ALMEIDA DC, KRAYCHETE DC. Dor lombar: uma abordagem diagnóstica. Revista Dor, São Paulo, 2017; 18(2): 173177.

2. ANDRADE SC, et al. Escola de Coluna: Revisão histórica e sua aplicação na lombalgia crônica. Revista Brasileira Reumatologia, São Paulo, 2005; 45(4): 224-228.

3. ANDRADE SC, et al. Escola de coluna para pacientes com lombalgia crônica inespecífica: benefícios da associação de exercícios e educação ao paciente. Acta Reumatologia, Portugal, 2008; 33(4), 443-450.

4. ARCANJO GN, et al.Percepção sobre qualidade de vida de mulheres participantes de oficinas educativas para dor na coluna. Revista Ciência \& Saúde Coletiva, 2008; 13(sup. 2): 2145-2154.

5. BARRETO JM, et al. O discurso do sujeito coletivo nas expectativas de mulheres com lombalgia participantes da escola de postura. Revista Psicologia Saúde e Debate, 2019; 5(2):112-124.

6. BARTZ PT, et al. Grupo da Coluna na Atenção Básica. Cadernos de Educação, Saúde e Fisioterapia, 2015; 2(3).

7. CHUNG TM. Escola de Coluna - experiência do Hospital das Clínicas da Universidade de São Paulo. Revista Acta Fisiatrica, São Paulo, 1996; 3(2): 13-17.

8. Di FÁBIO RP. Efficacy of Comprehensive Rehabilitation Program and Back School for Patients with Low Back Pain: A Meta Analysis. Physical Therapy, 1995; 75: 865-878.

9. FAIRBANK, JC, PYNSENT, PB. The oswestry disability index. Spine, New York, 2000; 25(22): $2940-2952$.

10. FERREIRA A, et al. Avaliação da qualidade de vida e incapacidade funcional lombar de estudantes universitários de educação física. Ciência Atual São José, Rio de Janeiro, 2017; 9(1): 1-15.

11. FERREIRA MS, NAVEGA MT. Efeitos de um programa de orientação para adultos com lombalgia. Acta Ortop Bras.,2010; 18(3): 127-131.

12. GAIDESKI F, et al. Avaliação da incapacidade funcional em indivíduos com dor lombar crônica. Revista Inspirar. movimento \& saúde, 2019;19(3).

13. HAEFFNER R, et al. Prevalência de lombalgia e fatores associados em trabalhadores de uma empresa agropecuária do sul do Brasil. Revista Brasileira Medicina do Trabalho, 2015;13(1): 35-42.

14. HELFENSTEIN MJ, et al. Lombalgia ocupacional. Revista Associação Medica Brasileira, São Paulo, 2010; 56(5): 583-589.

15. HOY D, et al. The epidemiology of a low back pain. Best Practice and Research Clinical Rheumatology., 2010; 24: 769-781.

16. IGUTI AM, et al. Dor nas costas em população adulta: estudo de base populacional em Campinas, São Paulo, Brasil. Cadernos Saúde Pública, 2015; 31(12): 2546-2558.

17. JUNIOR MH, et al. Lombalgia Ocupacional. Revista Associação Medica Brasileira, São Paulo, 2010; 56(5): $583-589$.

18. KORELO RIG, et al. Efeito de um programa cinesioterapêutico de grupo, aliado à escola de postura, na lombalgia crônica. Fisioterapia e Movimento, Curitiba, 2013; 26(2): 389-394.

19. MACEDO CSG, et al. Efeito do isostretching na resistência muscular de abdominais, glúteo máximo e extensores de tronco, incapacidade e dor em pacientes com lombalgia. Fisioterapia em Movimento, 2010; 23(1): 113-120.

20. MARTINS MRI, et al. A eficácia da conduta do grupo de postura em pacientes com lombalgia crônica. Revista Dor, 2010; 11(2):.105-110.

21. NASCIMENTO JM, et al. Métodos terapêuticos alternativos para o manejo da incapacidade da dor lombar crônica. Reme - Rev Min Enferm, 2015 19(1): 195-203. 
22. NOLL M, et al. Escolas posturais desenvolvidas no Brasil: revisão sobre os instrumentos de avaliação, as metodologias de intervenção e seus resultados. Revista Brasileira de Reumatologia, São Paulo, 2014; 54(1): 51-58.

23. OLIVEIRA ES, et al. Dor crônica sob a ótica dos pacientes da Escola de Postura da DMR HC FMUSP. Acta Fisiátrica, 2004; 11(1): 22-26.

24. PAKBAZ M, et al. Effectiveness of the back school program on the low back pain and functional disability of Iranian nurse. J Exerc Rehabil, 2019; 15(1): 134-138.

25. POLLI GB, et al. Atividade física e dor lombar em brasileiros: uma revisão sistemática- Physical activity and low back pain in Brazilians: a systematic review. Revista Brasileira Atividade Física e Saúde, 2018; 23:(47).

26. RODRIGUES GJT, GARCIA CA. Influência da escola da coluna em pacientes com lombalgia crônica. Revista Perquirere, Patos de Minas, 2017; 14(1): 190-199.

27. SAHIN N, et al. Effectiveness of back school for treatment of pain and functional disability in patients with chronic low back pain: a randomized controlled trial. J Rehabil Med, 2011; 43: 224-229.

28. SALVETTI MG, PIMENTA CAM. Dor crônica e a crença de auto-eficácia. Revista da Escola de Enfermagem da USP, 2007; 41(1): 135-140.

29. SALVETTI MG, et al. Incapacidade relacionada à dor lombar crônica: prevalência e fatores associados. Rev Esc Enferm, 2012; 46: 16-23.

30. SCHEUCHUK C, COMERLATO T. A Influência da Educação em Saúde sobre as respostas ao tratamento fisioterapêutico em indivíduos com lombalgia crônica. PERSPECTIVA, Erechim, 2017; 41(153): 97-106.

31. SHIRADO O, et al. A Novel Back School Using a Multidisciplinary Team Approach Featuring Quantitative Functional Evaluation and Therapeutic Exercises for Patients With Chronic Low Back Pain. Spine, 2005; 30(10): 1210-1225.

32. SILVA JCA, et al. Desempenho funcional e percepção da dor na lombalgia crônica após aplicação de um programa de Back School. SANARE, 2014; 13(1): 18-22.

33. SILVA PHB, et al. Perfil epidemiológico dos pacientes assistidos na clínica de fisioterapia traumato-ortopédica da prefeitura de Hidrolândia - Goiás. Revista Movimenta, 2013; 6(3): 520-529. 3.

34. SPONCHIADO P, CARVALHO AR. Descrição dos efeitos do protocolo Escola de Coluna Moderna em portadores de lombalgia crônica. Revista Fitness e Performance, 2007; 5(6): 283-288.

35. STEFANE S e MARINOVIC H. Dor lombar crônica: Intensidade de dor, incapacidade e qualidade de vida. Revista Acta Paulista de Enfermagem, São Paulo, 2013; 26(1): 14-20.

36. TOBO A, et al. Estudo do tratamento da lombalgia crônica por meio da Escola de Postura. Revista Acta Fisiatrica, São Paulo,2010; 17(3): 112-116.

37. TSUKIMOTO GR, et al. Avaliação longitudinal da Escola de Postura para dor lombar crônica através da aplicação dos questionários de Roland Morris e Short Form Health Survey (SF-36). Revista Acta Fisiátrica, São Paulo, 2006; 13(2): 63-69.

38. VALENÇA JBM, ALENCAR MCB. O afastamento do trabalho por dor lombar e as repercussões na saúde: velhas questões e desafios que continuam. Caderno Brasileiro de Terapia.Ocupacional, São Carlos, 2018; 26(1): 119-127.

39. VIGATTO R, et al. Development of a Brazilian Portuguese Version of the Oswestry Disability Index: Cross-Cultural Adaptation, Reliability, and Validity. Spine, 2007; 32(4): 481-486.

40. YANG EJ, et al. The effect of Back School integrated with Core strengthening in patients with chronic low-back pain. Am. J. Phys. Med. Rehabil, 2010; 89(9): 744-754. 